\title{
Upconversion solar cell measurements under real sunlight
}

\author{
Stefan Fischer ${ }^{1,2}$, Aruna Ivaturi ${ }^{3,4}$, Karl W. Krämer ${ }^{5}$, Peter Jakob², Rosa Martin-Rodriguez ${ }^{6,7}$, \\ Andries Meijerink ${ }^{6}$, Bryce Richards ${ }^{8}$, Jan Christoph Goldschmidt ${ }^{*}$ \\ ${ }^{1}$ Department of Materials Science and Engineering, Stanford University, Stanford, CA 94305, \\ USA \\ ${ }^{2}$ Fraunhofer Institute for Solar Energy Systems, Heidenhofstr. 2, 79110 Freiburg, Germany \\ ${ }^{3}$ WestCHEM, Department of Pure and Applied Chemistry, University of Strathclyde, Thomas \\ Graham Building, 295 Cathedral Street, Glasgow, G1 1XL, Scotland, UK \\ ${ }^{4}$ School of Engineering and Physical Sciences, Heriot-Watt University, EH14 4AS, Edinburgh, \\ United Kingdom \\ ${ }^{5}$ Department of Chemistry and Biochemistry, University of Bern, Freiestrasse 3, 3012 Bern, \\ Switzerland \\ ${ }^{6}$ Debye Institute for Nanomaterials Science, Department of Chemistry, Utrecht University, \\ Princetonplein 5, 3584 CC Utrecht, The Netherlands \\ ${ }^{7}$ QUIPRE Department, University of Cantabria, Avda. de Los Castros 46, 39005 Santander, \\ Spain \\ ${ }^{8}$ Institute of Microstructure Technology (IMT), Karlsruhe Institute of Technology, Hermann-von- \\ Helmholtz-Platz 1, 76344 Eggenstein-Leopoldshafen, Germany
}

Keywords:

Corresponding author: *jan.christoph.goldschmidt@fraunhofer.ise.de

\begin{abstract}
The main losses in solar cells result from the incomplete utilization of the solar spectrum. Via the addition of an upconverting layer to the rear side of a solar cell, the otherwise-unused subbandgap photons can be utilized. In this paper, we demonstrate an efficiency enhancement of a silicon solar cell under real sunlight due to upconversion of sub-bandgap photons. Sunlight was concentrated geometrically with a lens with a factor of up to 50 suns onto upconverter silicon solar cell devices. The upconverter solar cell devices (UCSCDs) were also measured indoors using a solar simulator. To correct for differences in the spectral distribution between real sunlight and the solar simulator a spectral mismatch correction is required and is especially important to properly predict the performance when a non-linear response (e.g. upconversion) is involved. By applying a spectral mismatch correction, good agreement between the solar
\end{abstract}


simulator measurements and the outdoor measurements using real sunlight was achieved. The method was tested on two different upconverter powders, $\beta$-NaYF 4 : $25 \% \mathrm{Er}^{3+}$ and $\mathrm{Gd}_{2} \mathrm{O}_{2} \mathrm{~S}$ : $10 \%$ $\mathrm{Er}^{3+}$, which were both embedded in a polymer. We determined additional photocurrents due to upconversion of $9.4 \mathrm{~mA} / \mathrm{cm}^{2}$ with $\beta-\mathrm{NaYF}_{4}$ and $8.2 \mathrm{~mA} / \mathrm{cm}^{2}$ with $\mathrm{Gd}_{2} \mathrm{O}_{2} \mathrm{~S}$ under 94-suns concentration. Our results show $i$ ) the applicability of measurements using standard solar cell characterization equipment for predicting the performance of non-linear solar devices, and ii) underline the importance of applying proper mismatch corrections for accurate prediction of the performance of such non-linear devices.

\section{Introduction}

Significant spectral losses are caused by the discrepancy between the discrete bandgap of the absorbing material in single junction solar cells and the very broad solar spectrum, as illustrated in Figure 1(a). Transmission losses occur for photons with less energy than the bandgap, as they do not carry enough energy to generate free carriers. However, these low energy photons can be transmitted through the solar cell, if the device is designed accordingly, to be harvested via an upconverter. An upconverter material can be applied to the rear side of the solar cell to convert two or more of the sub-bandgap photons into photons that possess more energy than the bandgap energy of the solar cell's absorber material. ${ }^{1-12}$ These upconverted photons can then be utilized by the solar cell, as illustrated in Figure 1(b). 

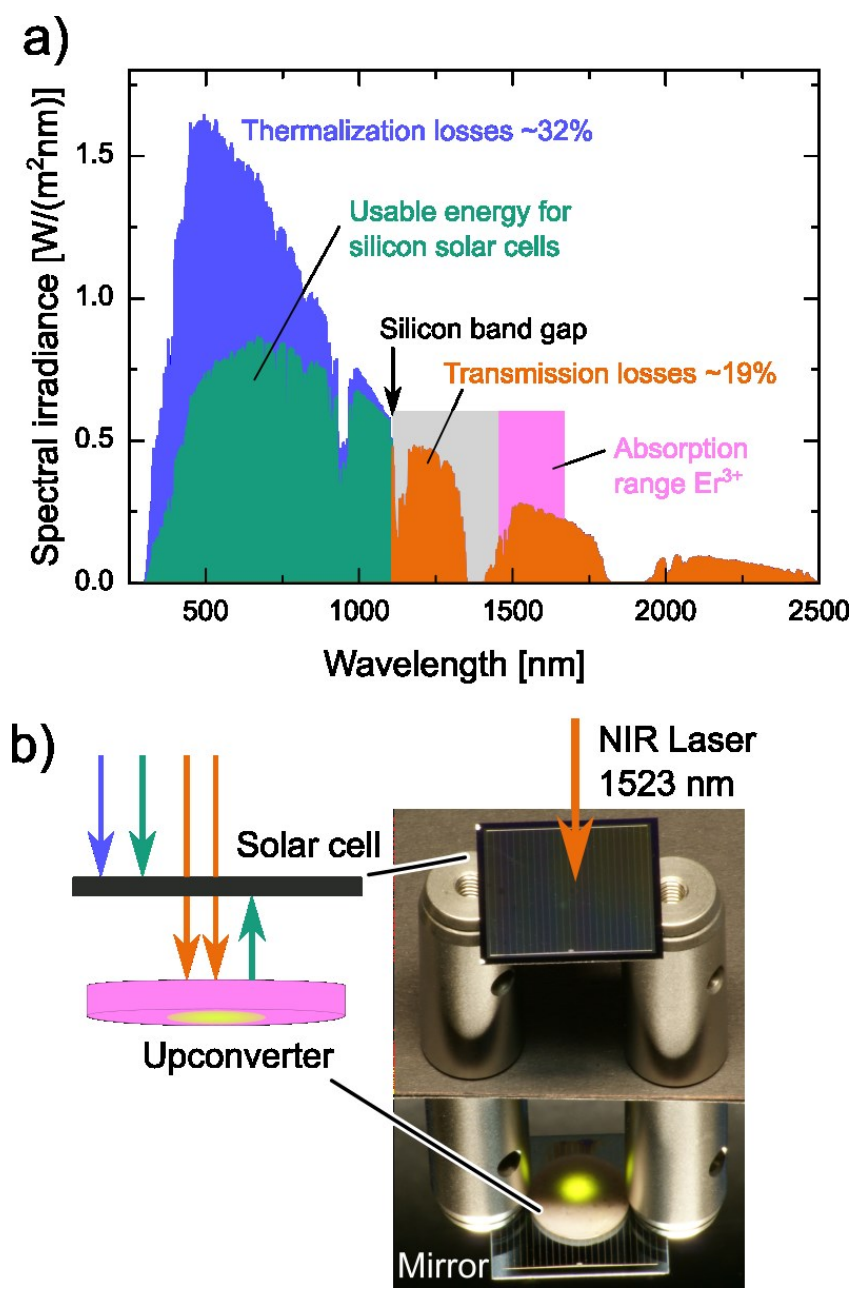

Figure 1. Spectral losses and upconversion to enhance solar cell performance. a) Air-mass 1.5 global (AM1.5G) standard solar spectrum with spectral losses indicated for a silicon solar cell. Around $50 \%$ of the energy from the sun reaching the earth's surface is not effectively utilized in silicon solar cells due to thermalization and transmission losses. b) An upconverter on the rear side of a solar cell can minimize the transmission losses by absorbing two or more sub-bandgap photons and emitting a photon that can be utilized by the solar cell. The image shows a $2 \times 2 \mathrm{~cm}^{2}$ bifacial silicon solar cell with an upconverter applied to the rear side. This upconverter solar cell device (UCSCD) is illuminated with $1523 \mathrm{~nm}$ laser light, which is transmitted through the solar cell and upconverted by the $\beta$-NaYF $4: \mathrm{Er}^{3+}$ upconverter material. The yellow-green upconversion is visible in the photograph by a reflection in a mirror.

The challenges associated with upconversion of sub-bandgap photons lie in both the solar cell technology and the upconverter material side. The concept of upconversion requires that the upconverter material needs to be placed at the rear side of the solar cell, which means that the solar cell has to be transparent for the sub-bandgap photons. Such bifacial solar cells are not the standard way of designing highly efficient solar cells, which typically requires a highly-reflective back mirror for good photon management and therefore high-power conversion efficiencies (PCE). In addition, also anti-reflection coating on the front and rear side of the solar cell need to 
be optimized to enable high transmittance of near-infrared (NIR, $\lambda>1000 \mathrm{~nm}$ ) photons. ${ }^{13}$ On the material side, the main challenges of suitable upconverter materials are the weak and narrow absorption range in the NIR, the non-linear nature of the upconversion efficiency, as well as the too low upconversion quantum yield (UCQY) values. Photo-chemical upconversion using triplettriplet annihilation addresses some of the above mentioned limitations but typical materials are not very photostable and limited to absorption wavelengths far below $1000 \mathrm{~nm}$ and emit usually in the visible spectrum. ${ }^{14,15}$ Currently, the most efficient upconverter materials for silicon solar cells are purely $\mathrm{Er}^{3+}$-doped inorganic crystals with absorption around $1500 \mathrm{~nm}$ and emission around $980 \mathrm{~nm}$. UCQY values above $10 \%$ have been reported for different microcrystalline and single crystal material system when using laser illumination with irradiances below $1 \mathrm{~W} / \mathrm{cm}^{2} .{ }^{11,16-}$ 20

The upconversion process in $\mathrm{Er}^{3+}$ is well described in literature $\mathrm{e}^{21-23}$ and the corresponding basic energy level diagram is shown in Figure 2a. In short, after ground state absorption (GSA) of photons with wavelengths of around $1500 \mathrm{~nm}\left({ }^{4} I_{15 / 2} \rightarrow{ }^{4} I_{13 / 2}\right)$ the excitation energy may hop around in the crystal via energy transfer from $\mathrm{Er}^{3+}$ ion to $\mathrm{Er}^{3+}$ ion. Energy transfer upconversion (ETU) occurs if two excited $\mathrm{Er}^{3+}$ ions in the ${ }^{4} \mathrm{I}_{13 / 2}$ energy level are close to each other, resulting in one ion relaxing to the ground state ${ }^{4} I_{15 / 2}$ while the other one is promoted to the higher excited state ${ }^{4} \mathrm{I}_{9 / 2}$. Via multi-phonon relaxation (MPR), the $\mathrm{Er}^{3+}$ ion relaxes from the ${ }^{4} \mathrm{I}_{9 / 2}$ to the ${ }^{4} \mathrm{I}_{11 / 2}$ state and the dominant emission around $980 \mathrm{~nm}$ occurs by spontaneous emission from ${ }^{4} \mathrm{I}_{11 / 2} \rightarrow{ }^{4} \mathrm{I}_{15 / 2}$. A typical emission spectrum of $\mathrm{Er}^{3+}$-doped upconverter materials under ${ }^{4} \mathrm{I}_{15 / 2} \rightarrow{ }^{4} \mathrm{I}_{13 / 2}$ excitation is shown in Figure $2 \mathrm{~b}$. UCQY values of the upconverter powders $\beta$-NaYF $4: 25 \% \mathrm{Er}^{3+}$ and $\mathrm{Gd}_{2} \mathrm{O}_{2} \mathrm{~S}: 10 \% \mathrm{Er}^{3+}$ of $7.5 \%$ and $12.0 \%$, respectively, have been measured using laser irradiation with $0.11 \mathrm{~W} / \mathrm{cm}^{2}\left(1100 \mathrm{~W} / \mathrm{m}^{2}\right) .{ }^{19}$ 


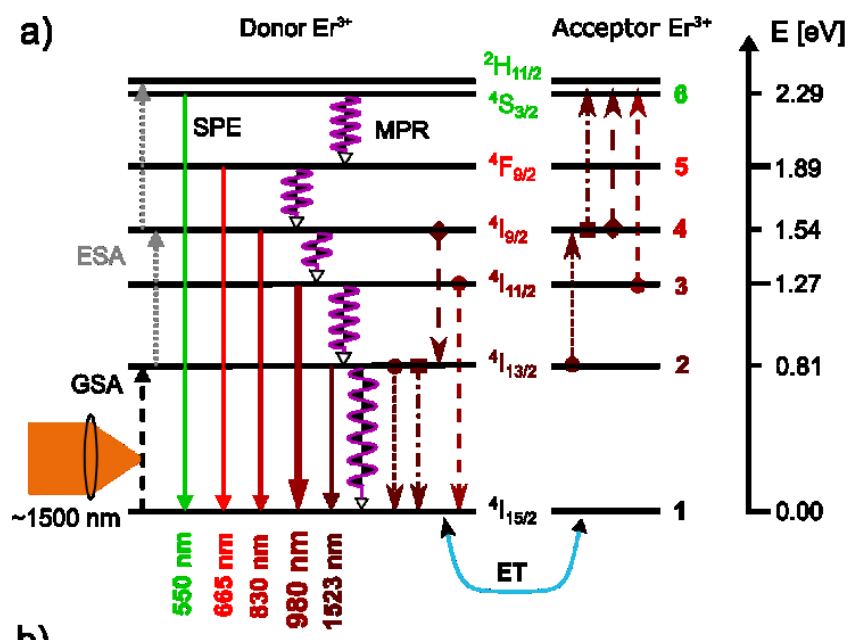

b)

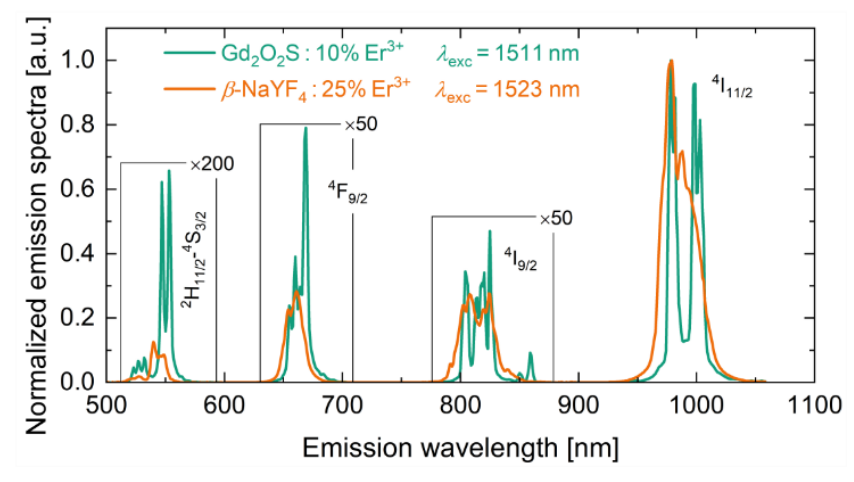

Figure 2. Upconversion mechanism. a) Energy level diagram of $\mathrm{Er}^{3+}$ and illustration of processes involved in the upconversion mechanism. Ground state absorption (GSA) is followed by energy transfer upconversion (ETU) or with less probability excited state absorption (ESA). Typically, after multi-phonon relaxation (MPR) spontaneous emission (SPE) from the ${ }^{4} I_{11 / 2}$-Level occurs, resulting in the dominating upconversion emission at around $980 \mathrm{~nm}$. b) Upconversion emission spectrum under ${ }^{4} I_{15 / 2} \rightarrow{ }^{4} I_{13 / 2}$ laser excitation around $\sim 1500 \mathrm{~nm}$ using an irradiance of $0.11 \mathrm{~W} / \mathrm{cm}^{2}$ for two different upconverter materials, $\mathrm{Gd}_{2} \mathrm{O}_{2} \mathrm{~S}: 10 \% \mathrm{Er}^{3+}$ and $\beta$-NaYF $4: 25 \% \mathrm{Er}^{3+}$.

In the past, we have demonstrated an enhanced performance of bifacial silicon solar cells with $\mathrm{Er}^{3+}$-doped $\beta$-NaYF 4 and $\mathrm{Gd}_{2} \mathrm{O}_{2} \mathrm{~S}$ upconverters attached on the rear side of solar cell using laser illumination ${ }^{6,24-26}$, broad-band excitation from a halogen lamp ${ }^{25,26}$, and concentrated light of a solar simulator ${ }^{24,25}$. To assess the suitability of an upconverter material for the application in photovoltaics, the material and UCSCDs should be characterized using a spectrally broad excitation source, which corresponds to the broad solar spectrum. Besides our previous works there is only a handful of this kind of characterization reported in literature ${ }^{10,27-29}$ The highest performance enhancement due to upconversion in silicon solar cells was reported using a $\mathrm{BaY}_{2} \mathrm{~F}_{8}$ single crystal doped with $30 \% \mathrm{Er}^{3+} .{ }^{11}$ An additional current of $17.2 \mathrm{~mA} / \mathrm{cm}^{2}$ was achieved under solar radiation concentrated geometrically by a factor of 94 , which is typically referred to as a concentration of 94 suns. This constitutes relative enhancement of the solar cell's short circuit current density by $0.55 \%$. The measurements were performed using a solar simulator, 
which is designed to mimic the air-mass 1.5 global (AM1.5G) standard solar spectrum. However, the actual spectrum of a solar simulator is far from ideal in the wavelengths region where the upconverter operates of roughly $1400 \mathrm{~nm}$ to $1650 \mathrm{~nm}$ (see also Figure 5). Therefore, a spectral mismatch correction has to be applied addressing the mismatch of the spectrum from the solar simulator setup and the AM1.5G ${ }^{11,26}$ However, the accuracy of this mismatch correction has never been demonstrated in the context of upconverter solar cell devices by comparison to real sunlight measurements. Therefore, in this paper we report for the first time upconversion enhanced solar cell performance in outdoor measurements using sunlight with concentrations up to 50 suns. In addition, we probe our spectral mismatch correction method for these highly nonlinear systems by comparing the measurements outdoor at real insolation conditions with sunlight to the ones using a solar simulator indoors with applied mismatch correction. This step is important because it validates the use of standardized measurement tools to predict the performance of UCSCDs in real world.

\section{Experimental Section}

Upconverter silicon solar cell devices were fabricated as described in previous literature. ${ }^{11,25,26}$ Briefly, we used bifacial silicon solar cells with planar front and rear side for maximum transmission of sub-bandgap photons. ${ }^{13,30}$ While the anti-reflection coatings were optimized for sub-bandgap photon transmittance, the devices also exhibited PCEs above $16 \%$ when measured using a solar simulator and standard measurement conditions. ${ }^{13}$ These PCE values are fairly good considering the lack of a front-side texturing and a rear reflector. The microcrystalline upconverter materials $\beta$-NaYF $4: 25 \% \mathrm{Er}^{3+}$ and $\mathrm{Gd}_{2} \mathrm{O}_{2} \mathrm{~S}: 10 \% \mathrm{Er}^{3+}$ were previously identified as promising candidates for solar energy harvest of sub-bandgap photons. ${ }^{17,19}$ Therefore, we explore these materials further in this study and embedded the powders in the polymer perfluorocyclobutan (PFCB) in disks with diameters of $12.6 \mathrm{~mm}$ and thickness of roughly $1 \mathrm{~mm} .{ }^{16}$ The ratio of upconverter to polymer was $75.7 \mathrm{w} / \mathrm{w} \%$ for $\beta-\mathrm{NaYF}_{4}: 25 \% \mathrm{Er}^{3+}$ and 84.9 w/w\% for $\mathrm{Gd}_{2} \mathrm{O}_{2} \mathrm{~S}: 10 \% \mathrm{Er}^{3+}$.

These upconverter disks were attached sequentially to the same solar cell with an index matching liquid (IML, Immersion oil, Type 300, Cargille). For easier handling the bifacial silicon solar cell was soldered onto a copper frame to form a rear contact. The front contacts were wire bonded onto larger contact pads. A reflector - that was made of highly reflective porous polytetrafluoroethylene (PTFE) - was placed behind the UCSCDs, as illustrated in Figure 3a. The indoor measurements were carried out as described in the literature. ${ }^{11,25}$ For easier handling and mounting the PTFE reflector was further embedded in a larger copper block for the outdoor measurements. This copper block was mounted on a solar tracker following the path of the sun to measure the short-circuit current in UCSCDs with sunlight of normal incidence during the whole measurement period. To determine precisely the additional current due to upconversion, 
in both experiments, we conducted reference measurements with disks of un-doped $\beta$-NaYF 4 and $\mathrm{Gd}_{2} \mathrm{O}_{2} \mathrm{~S}$ powders mixed with $\mathrm{PFCB}$, which only scatter the light without upconverting it.

The efficiency of solar cells is commonly measured under solar simulators. In solar simulators, different lamps in combination with lenses, mirrors, and filters are used to generate an artificial solar spectrum that is supposedly as close as possible to the AM1.5G. These are the most realistic approaches for investigating the performances of solar cells that are available under well-controlled and reproducible conditions. However, spectral mismatch corrections have to be applied to predict the performance of the solar cells under real operation. This is a standard procedure in photovoltaic calibration labs and when measuring solar cells. However, the nonlinear nature of the upconversion process for NIR light (compared to the linear response of the solar cell) complicates spectral mismatch corrections. In this work, we experimentally evaluate the spectral mismatch correction for highly-nonlinear materials that we developed in our previous studies. ${ }^{11,26}$

Here, we measured UCSCDs under the concentrated radiation from a solar simulator (Wacom, WXS 150S-10, class A) with a Xe-lamp and in an outdoor experiment using concentrated sunlight. In these measurements, a Fresnel lens was used to focus either the light from the solar simulator or the light from the sun onto upconverter solar cell devices. The silicone Fresnel lens features a focal length of approximately $168 \mathrm{~mm}$ and a collecting area $A_{\text {lens }}$ of $100 \times 100 \mathrm{~mm}^{2}$. The distance between the lens and the upconverter solar cell device $h_{\text {lens }}$ can be adjusted with a translation stage. Changing $h_{\text {lens }}$ alters the illuminated area on the solar cell $A_{\text {spot, }}$ and consequently also the concentration of the radiation onto the sample.

a)

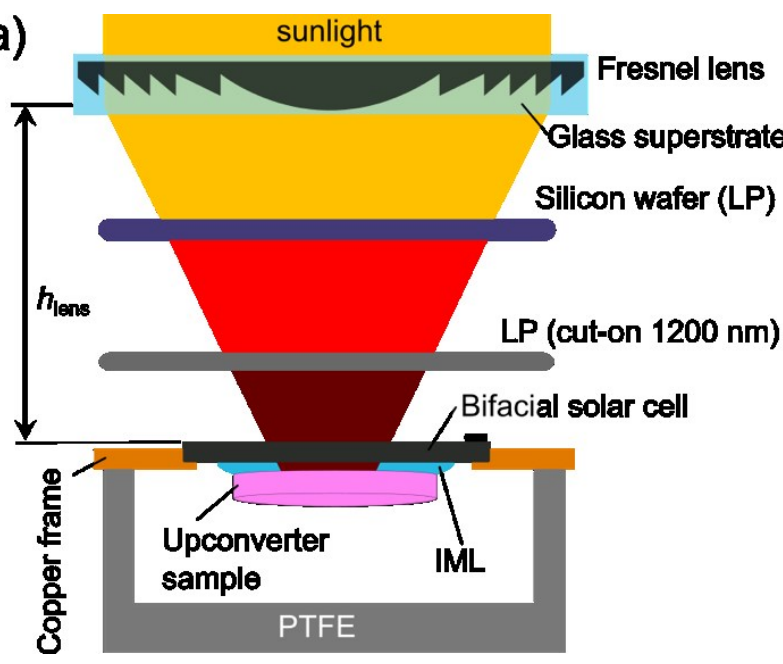

b)

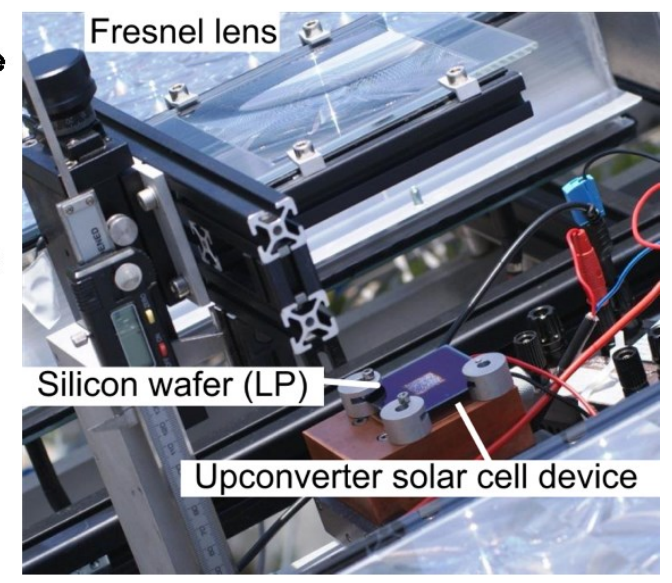

Figure 3. Schematic and photograph of the UCSCD and its characterization using solar radiation. a) The height of the lens above the UCSCD is altered thus changing the area of the light spot $A_{\text {spot }}$ and consequently the concentration of the solar light on the sample. Twool long pass (LP) filters were used to block the fraction of the light that can be directly used by the silicon solar cell. The upconverter samples are attached by adhesion using an index matching 
liquid (IML). b) Picture of the setup for the outdoor measurements of upconverter solar cell devices. The rectangular light spot from the lens can be seen on the silicon wafer serving as a LP filter.

A scheme of the setup is shown in Figure 3 together with a photograph of the outdoor experiment. Two filters were applied above the solar cell to block photons that otherwise could have been utilized directly by the silicon solar cell. This way the signal-to-noise ratio could be improved. Firstly, a polished monocrystalline $750 \mu \mathrm{m}$ thick silicon wafer, which was coated on both sides with $120 \mathrm{~nm}$ magnesium difluoride $\left(\mathrm{MgF}_{2}\right)$ on top of $120 \mathrm{~nm}$ titanium dioxide $\left(\mathrm{TiO}_{2}\right)$ to enhance the transmittance of infrared photons. Secondly, a long pass filter (Edmund Optics, High Performance OD 4) with a cut-on wavelength of $1200 \mathrm{~nm}$. The second filter served as an additional NIR filter for photons close to the band gap of silicon, which are transmitted through the silicon filter and would have been utilized by the solar cell.

The Fresnel lens employed in this work was produced at Fraunhofer ISE by molding silicone. Because upconversion is a non-linear process, it is critical to know the exact irradiance or sunlight concentration ratio illuminating the sample. Therefore, the optical properties of the Fresnel lens were characterized in detail with the setup described in literature. ${ }^{31}$ In short, the light of a red light emitting diodes (LEDs) at $622 \mathrm{~nm}$ was modified with an collimator lens to mimic the divergence of sunlight. This light spot was concentrated by the Fresnel lens and measured with a cooled CCD sensor. The distance between the CCD sensor and the Fresnel lens can be precisely changed with a motorized translation stage. Figures $4 a-c$ show pictures of the light spot for different distances between the Fresnel lens and the CCD sensor. Around the focal plane at $h_{\text {lens }}=168 \mathrm{~mm}$ a circular spot was determined. For shorter distances, the light spot becomes rectangular and the irradiance distribution more inhomogeneous. The pixels with the largest irradiance were counted until $90 \%$ of the total light irradiance measured by the CCD sensor was obtained. From the dimensions of the single pixels and the number of counted pixels, the area of the light spot $A_{\text {spot }}$ was calculated. The values of $A_{\text {spot }}$ as a function of $h_{\text {lens }}$ are shown as symbols in Figure $4 \mathrm{~d}$. The homogeneity of the irradiance distribution of the area $A_{\text {spot }}$ was determined by the standard deviation of the irradiance's histogram, which was measured throughout the area $A_{\text {spot. }}$ A standard deviation of around $2.7 \%$ was found. Furthermore, we calculated a value of $7.4 \%$ for the difference between the maximum intensity of a pixel and the mean value for all pixels contributing to $A_{\text {spot. }}$ Therefore, we can conclude a fairly homogeneous irradiance distribution of the light spot $A_{\text {spot, }}$ which is important to calculate the correct concentration of the incident illumination. The optical efficiency of the Fresnel lens $\eta_{\text {lens }}$ is defined as the fraction of the total irradiance determined by the CCD sensor in the focal plane divided by the total irradiance on the Fresnel lens' entrance aperture. An optical efficiency $\eta_{\text {lens }}$ of $87.2 \pm 2.0 \%$ was obtained. The losses are caused by reflection (roughly $8 \%$ ) at the two glass surfaces of the Fresnel lens, and by scattering and absorption. 
a)

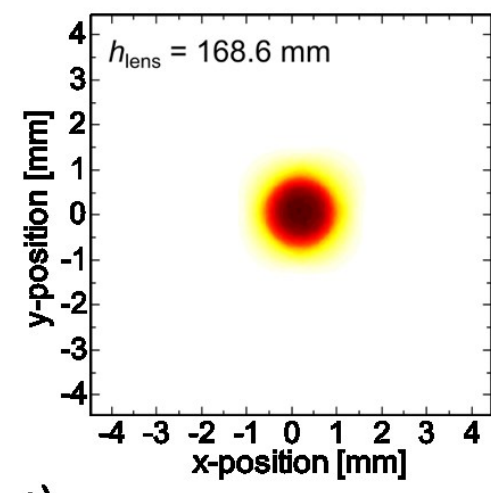

c)

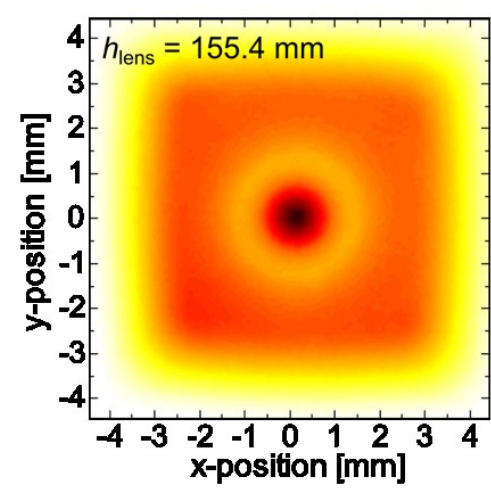

b)
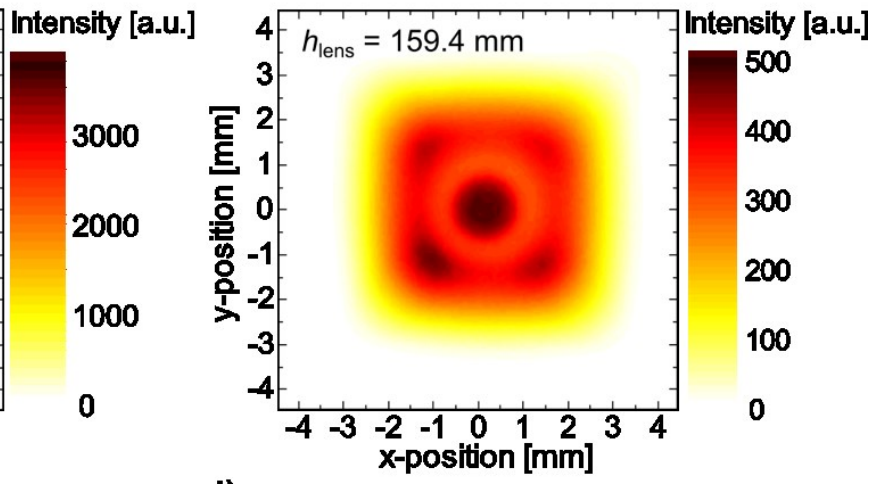

d)

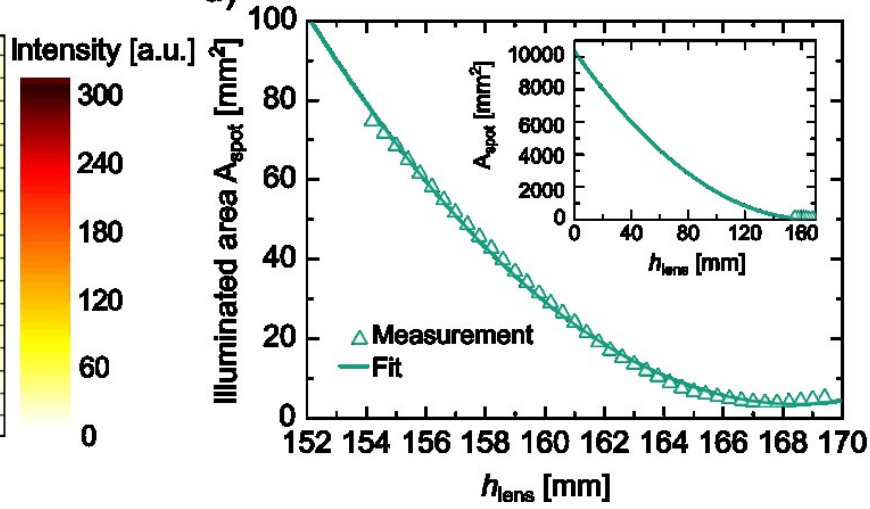

Figure 4. Characterization of the Fresnel lens to determine the concentration of the solar radiation on the UCSCDs. a)-c) Images of the illuminated area and irradiance distribution for various distances between the lens and a CCD detector $\left(h_{\text {lens }}\right)$. d) The function defined in Equation 1 was fitted to the data in order to parameterize the illuminated area $A_{\text {spot }}$ by $h_{\text {lens }}$ and further used to extrapolate the measurements to the larger areas that are used in this work.

To extrapolate the data, the area of the light spot as a function of $h_{\text {lens }}$ was parameterized by

$$
A_{\text {spot }}\left(h_{\text {lens }}\right)=c_{\text {lens }}\left(h_{\text {lens }}-h_{0}\right)^{2}+A_{0}
$$

with the constant $c_{\text {lens, }}$, the focal length $h_{0}$, and the light spot area at the focal length $A_{0}$. The fitted function agrees well with the data. The parameters obtained are $c_{\text {lens }}=0.363 \pm 0.008$, $h_{0}=168.4 \pm 0.2 \mathrm{~mm}$, and $A_{0}=3.6 \pm 0.4 \mathrm{~mm}^{2}$. An indicator of the good fit quality is the minimal (3.1\%) difference between the lens area and $A_{\text {spot }}$ for an extrapolation to $h_{\text {lens }}=0 \mathrm{~mm}$. Using Equation $1, A_{\text {spot }}$ was calculated from the measured $h_{\text {lens }}$ and used to determine the solar concentration factor $C$ of the AM1.5G standard solar spectrum

$$
C\left(A_{\text {spot }}\right)=\frac{\eta_{\text {lens }}}{c_{\text {mismatch }}} \frac{A_{\text {lens }}}{A_{\text {spot }}} \frac{I_{\mathrm{DNI}}}{I_{\mathrm{AM} 1.5}} .
$$


One has to note, however, that the parametrized spot size $A_{\text {spot }}(h)$ is based on monochromatic measurements (i.e. $622 \mathrm{~nm}$ ), only. Nevertheless, we use this spot size as approximation also for the long wavelength side of the polychromatic irradiance we are interested in during the upconversion experiments, as typically chromatic aberration of a Fresnel lens is critical only for short wavelength.

For the indoor measurements using the solar simulator, we need to consider the spectral mismatch correction factor

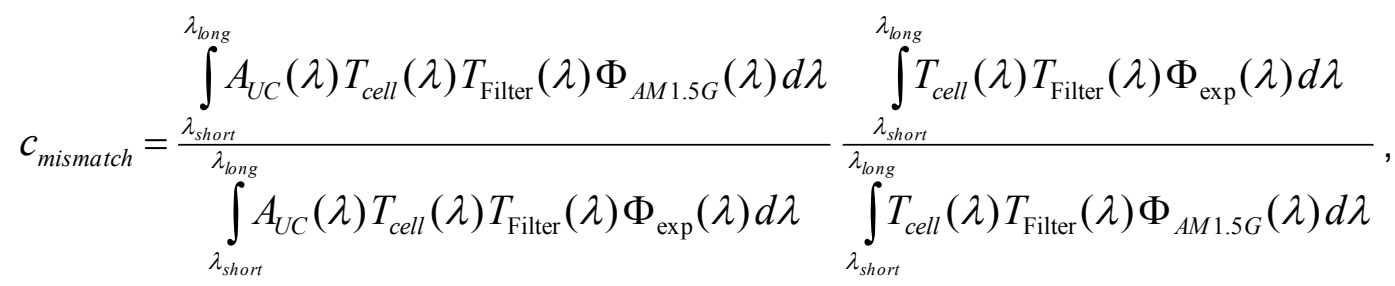

where $A_{u c}$ is the normalized absorption spectrum of the upconverter, $T_{\text {cell }}$ is the transmittance of the solar cell, $T_{\text {Fiter }}$ is the transmittance of all the optical components and filters used, and the photon flux densities of the standard solar spectrum $\Phi_{\mathrm{AM} 1.5 \mathrm{G}}$ as well as the experimental spectrum of the solar simulator $\Phi_{\text {exp }}$. The integration limits are determined by the considered spectral range which is $1400 \mathrm{~nm}$ to $1650 \mathrm{~nm}$ in our case. The absorption spectra and photon flux densities used to calculate $c_{\text {mismatch }}$ are shown in Figure 5 . We determined $c_{\text {mismatch }}$ values of 1.10 for $\beta$-NaYF 4 and 1.14 for $\mathrm{Gd}_{2} \mathrm{O}_{2} \mathrm{~S}$ using optical components used in this study. The outdoor measurements where conducted at a rooftop measurement platform at Fraunhofer ISE in Freiburg, Germany (48 $\left.00^{\prime} 34.3^{\prime \prime} N, 7^{\circ} 49^{\prime} 57.6^{\prime \prime} \mathrm{E}\right)$, in the first week of July 2013 with clear sky. The Fresnel lens only concentrates the direct radiation. However, because of the low scattering of infrared light, there is no significant difference between the direct and the global solar spectrum in the wavelength range around $1500 \mathrm{~nm}$. Nevertheless, the direct normal irradiance (DNI) from the sun fluctuated during the measurements due to a few cirrocumulus clouds and the varying position of the sun (air mass varied between roughly 1.1 and 1.7). To make the data comparable the concentration factor is corrected by the ratio of the $\mathrm{DNI}\left(\mathrm{I}_{\mathrm{DNI}}\right)$, which was measured during each individual measurement, and the irradiance in the AM1.5G $\left(I_{\mathrm{AM} 1.5 \mathrm{G}}=1000 \mathrm{~W} / \mathrm{cm}^{2}\right)$. We measured the DNI with a pyrheliometer (Kipp \& Zonen) at the same time that the short-circuit current of the UCSCDs was measured. The DNI varied in the course of the measurements between $756 \mathrm{~W} / \mathrm{m}^{2}$ to $890 \mathrm{~W} / \mathrm{m}^{2}$. Despite the significant changes in $\mathrm{DNI}$, the spectral shape relevant for the spectral mismatch correction does not change severely during the measurement time around noon. In consequence, no mismatch correction was applied to the outdoor measurements $\left(c_{\text {mismatch }}=1\right)$ and the results were compared to the solar simulator measurements corrected for the mismatch to the AM1.5G spectrum. 


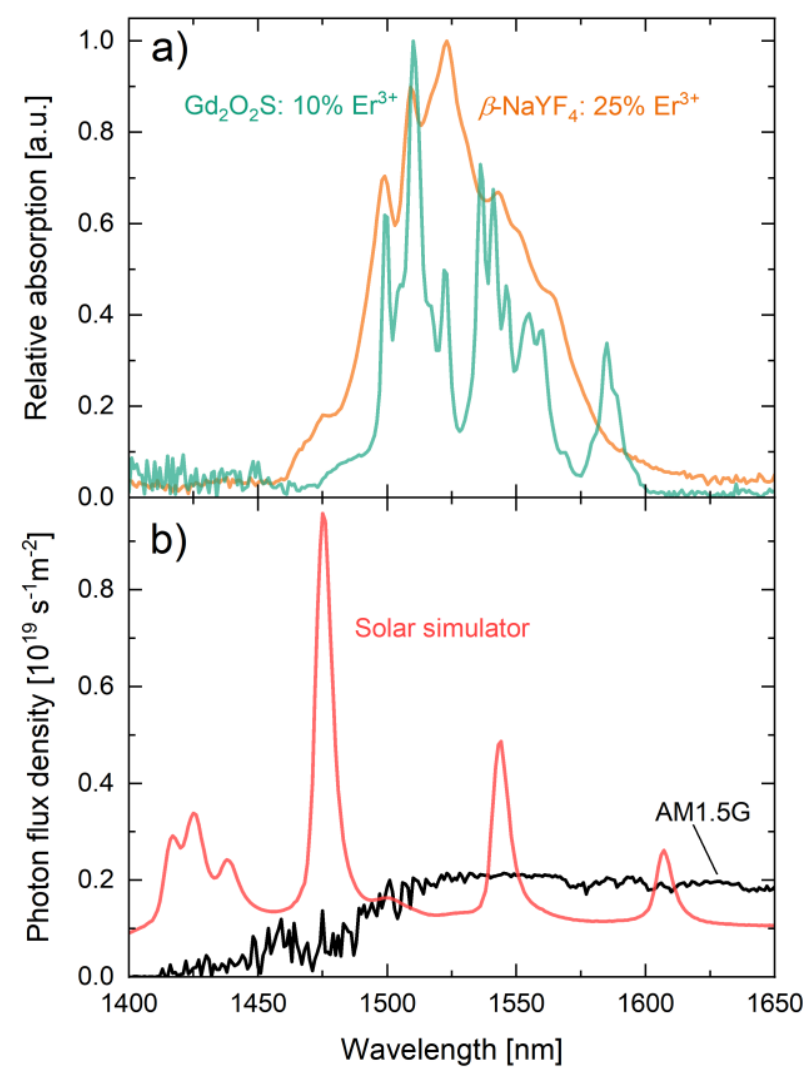

Figure 5. Considered spectra for the spectral mismatch correction. a) Difference in the normalized absorption spectra of the $\mathrm{Er}^{3+}{ }^{4} \mathrm{I}_{15 / 2}$ to ${ }^{4} \mathrm{I}_{13 / 2}$ transition in the host lattices $\beta$-NaYF $\mathrm{F}_{4}$ and $\mathrm{Gd}_{2} \mathrm{O}_{2} \mathrm{~S}$. b) Illustration of the need for a spectral mismatch correction due to the differences between the photon flux density provided by the solar simulator and the standard AM1.5G solar spectrum. The $c_{\text {mismatch }}$ values for the UCSCDs used in this study are 1.10 for the upconverter with $\beta$-NaYF 4 host lattice and 1.14 for $\mathrm{Gd}_{2} \mathrm{O}_{2} \mathrm{~S}$, respectively. Note, the solar spectrum is blownup to only show the spectral range relevant for the spectral mismatch correction of the $\mathrm{Er}^{3+}{ }^{4} I_{15 / 2}$ to ${ }^{4} I_{13 / 2}$ transition. The sharp lines in the solar simulator spectrum originate from the Xe lamp.

The additional short-circuit current density due to upconversion of sub-bandgap photons $\Delta j$ sc,uc can be calculated by

$$
\Delta j_{\mathrm{SC}, \mathrm{UC}}(C)=\frac{i_{\mathrm{SC}, \mathrm{UC}}(C)-i_{\mathrm{SC}, \mathrm{ref}}(C)}{A_{\mathrm{spot}}\left(h_{\text {lens }}\right)} c_{\text {mismatch }}
$$

using the short-circuit currents measured for the device with an upconverter $i_{\mathrm{sc}, \mathrm{uc}}$, as well as for a sample with the pure host material without any upconversion that mimicks the reflection of the sample $i_{\text {sc,ref. }}$ Here, for $A_{\text {spot }}$ values larger than the actual solar cell area $A_{\text {cell }}$ of $400 \mathrm{~mm}^{2}, A_{\text {cell }}$ is used instead of $A_{\text {spot }}$ to calculate $\Delta j$ sc,uc.

The main error of the solar concentration factor originates from the determination of $A_{\text {spot, }}$ or rather, from the precise measurement of $h_{\text {lens. }}$ For a typical uncertainty of $0.25 \mathrm{~mm}$ of the $h_{\text {lens }}$ 
measurement, the errors for $A_{\text {spot }}$ range from roughly $7.8 \%$ for $h_{\text {lens }}=130 \mathrm{~mm}$ up to $9.9 \%$ for $h_{\text {lens }}=160 \mathrm{~mm}$. The errors in the solar concentration factor $C$ and in the $\Delta j_{\mathrm{sc}, u \mathrm{u}}$ are higher than the ones for $A_{\text {spot }}$ because of the additional errors in the current measurements, the spectral

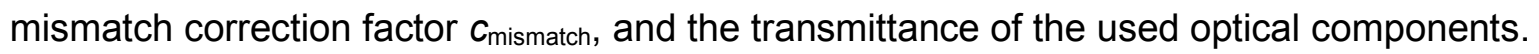

\section{Results and Discussion}

The $\Delta j \mathrm{sc}, \mathrm{uc}$ values determined with the solar simulator with and without spectral mismatch correction are shown in Figure 6. The spectral mismatch correction results in higher current densities at lower solar concentrations for both the $\beta-\mathrm{NaYF}_{4}$ and $\mathrm{Gd}_{2} \mathrm{O}_{2} \mathrm{~S}$ host matrix. Therefore, without spectral mismatch correction the efficiency enhancement potential of these materials is underestimated given the specific set of solar simulator and used materials. This agrees with our previous results of these materials but is in contrast to other materials ${ }^{26}$, such as the single crystal $\mathrm{BaY}_{2} \mathrm{~F}_{8}$ for which the correction yields lower current densities at higher solar concentrations. ${ }^{11}$ It is important to point out that the $c_{\text {mismatch }}$ values vary with different solar simulator spectra in the NIR and consequently vary with different solar simulator models. In our case, the strong peaks from the Xe lamp mainly determine the spectral mismatch correction values.

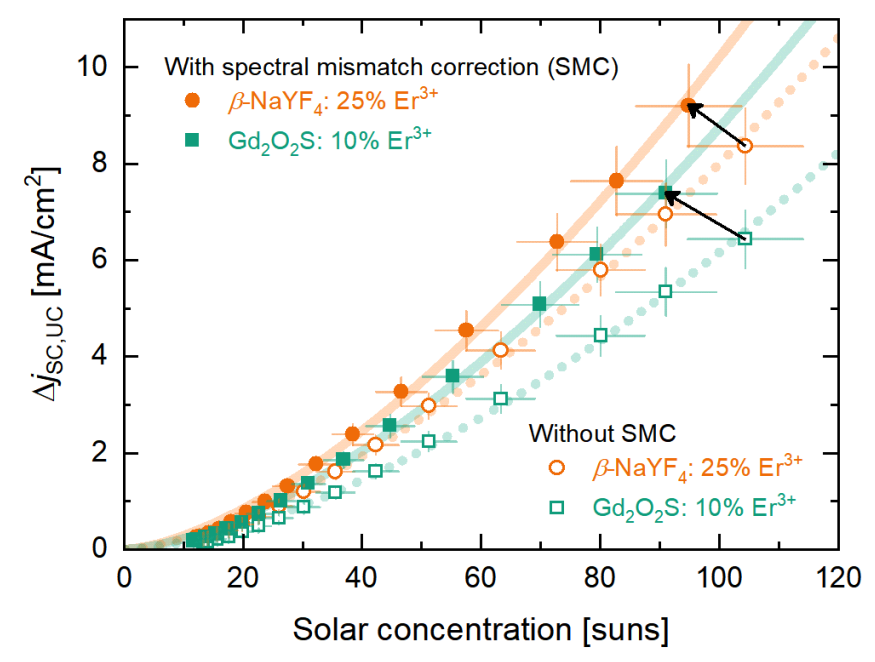

Figure 6. Increase of the short-circuit current density due to upconversion of sub-bandgap photons $\Delta j$ sc,uc. Due to the spectral mismatch correction, we determined higher $\Delta j \mathrm{jc}, u c$ values at lower solar concentration factors which is indicated by the black arrows. This trend is mostly caused by the strong peaks from the Xe lamp in the solar simulator spectrum as shown in Figure 5 which do not overlap significantly with the absorption spectrum of either one of the upconverter samples. Consequently, without mismatch correction the solar concentration is overestimated. The lines are fits to the data using Equation 5.

The experimental data is well described by a power law 


$$
\Delta j_{\mathrm{SC}, \mathrm{UC}}(C)=a \cdot C^{b}
$$

with the fitting parameters $a$ and $b$. The parameters for the spectral mismatch correction modified data are $a=0.0081 \mathrm{~mA} / \mathrm{cm}^{2}$ and $b=1.55$ for the $\beta-\mathrm{NaYF}_{4}$ and $a=0.0059 \mathrm{~mA} / \mathrm{cm}^{2}$ and $b=1.59$ for the $\mathrm{Gd}_{2} \mathrm{O}_{2} \mathrm{~S}$ upconverter sample. For an ideal multi-photon process the exponent should be equal to the number of photons involved. However, we can see that the device as a whole does not behave as an ideal upconverter system for which we would expect a parameter $b=2$. Nevertheless, an exponent higher than 1 and the lack of any saturation effect suggests that higher efficiency enhancements are achievable at higher solar concentrations.

We used the fitting parameters to calculate the $\Delta j_{\mathrm{sc}, u c}$ values at 94 suns for which the highest values are reported for the currently most efficient UCSCD. The UCSCDs reach $\Delta j_{\text {sc,uc }}$ values of $9.4 \mathrm{~mA} / \mathrm{cm}^{2}$ with $\beta$-NaYF 4 and $8.2 \mathrm{~mA} / \mathrm{cm}^{2}$ with $\mathrm{Gd}_{2} \mathrm{O}_{2} \mathrm{~S}$. These values are much lower compared to the ones obtained for $\mathrm{BaY}_{2} \mathrm{~F}_{8}$ of $17.2 \mathrm{~mA} / \mathrm{cm}^{2}$. One important reason for the much better performance of the $\mathrm{BaY}_{2} \mathrm{~F}_{8}$ single crystals is the absence of scattering in combination with the higher absorption by the single crystal sample. While we found that the $\mathrm{BaY}_{2} \mathrm{~F}_{8}$ samples absorbs $94 \%$ at the peak in the absorption spectrum at $1520 \mathrm{~nm}$, the $\beta-\mathrm{NaYF}_{4}$ and $\mathrm{Gd}_{2} \mathrm{O}_{2} \mathrm{~S}$ samples absorbed only $86 \%$ and $69 \%$ at their peak absorption at $1523 \mathrm{~nm}$ and $1511 \mathrm{~nm}$, respectively. ${ }^{26,32}$ Other parameters to consider in addition to the absorptance at a single wavelength are the shape and the width of the absorption spectrum. In this category, the $\beta$ $\mathrm{NaYF}_{4}$ and $\mathrm{BaY}_{2} \mathrm{~F}_{8}$ outperform the $\mathrm{Gd}_{2} \mathrm{O}_{2} \mathrm{~S} .{ }^{11,26}$

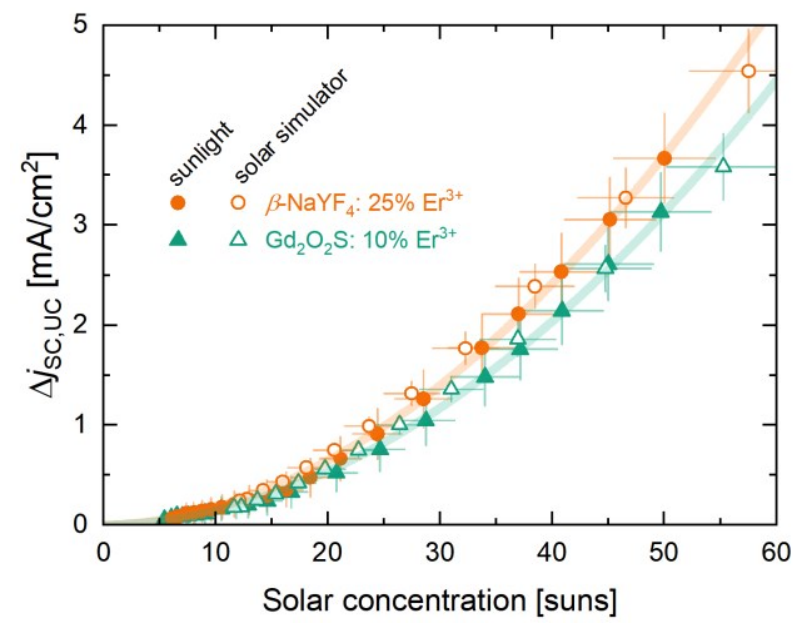

Figure 7. Comparison of $\Delta j \mathrm{sc}, u \mathrm{c}$ measurements using sunlight and a solar simulator after applying a spectral mismatch correction. The results with spectral mismatch correction show very similar values to the measurements performed using real sunlight corrected by the actual DNI on the UCSCD. Therefore, we conclude that spectral mismatch correction corrected measurements predict the performance of highly non-linear solar devices very well.

Next, we measured the same UCSCDs with the same Fresnel lenses outside with sunlight as described in the Experimental Section. The $\Delta j s c, u c$ values are in good agreement with the results 
from solar simulator measurements with spectral mismatch correction applied as shown in Figure 7. Therefore, we can conclude that measurement using standard equipment, such as solar simulators, can be used to predict the performance of highly non-linear solar devices, provided a correct mismatch correction is applied.

What is also clear from the measurement results, however, is that for a future application in photovoltaics, we still need to work on enhancing the UCQY and also decrease the irradiance necessary to achieve such high UCQY values. Our work revealed the need for upconverter materials that exhibit a broad and strong absorption spectrum. This is highlighted by the fact that although higher UCQY values have been achieved for $\mathrm{Gd}_{2} \mathrm{O}_{2} \mathrm{~S}$ under monochromatic excitation the use of the $\beta$-NaYF 4 host matrix results in better UCSCD performance when broadband or solar radiation is used. In order to use the complete sub-bandgap region of the solar spectrum we need to couple upconverter materials to broadband downshifting materials as suggested before. ${ }^{10,33}$ This is one very promising approach to overcome the fairly narrow absorption range characteristic to the lanthanide elements used to build efficient NIR upconverter materials and one key step towards more efficient solar energy harvesting using upconverter materials.

\section{Conclusion \& Outlook}

We fabricated upconverter solar cell devices for testing in a controlled environment using a solar simulator as well as in an outdoor experiment using sunlight with concentrations of up to around 50 suns. We used the same bifacial silicon solar cell with four different samples attached to the rear side, which are the upconverting powders $\mathrm{Gd}_{2} \mathrm{O}_{2} \mathrm{~S}: 10 \% \mathrm{Er}^{3+}$ and $\beta-\mathrm{NaYF}_{4}: 25 \% \mathrm{Er}^{3+}$ as well as the un-doped powders $\mathrm{Gd}_{2} \mathrm{O}_{2} \mathrm{~S}$ and $\beta$-NaYF 4 for reference measurements. The indoor measurements using a solar simulator showed a non-linear increase in the additional shortcircuit current density $\Delta j$ sc,uc of the upconverter solar cell device (UCSCD) with solar concentration. We applied a spectral mismatch correction to correct for the differences between the standard solar spectrum and the spectrum of the solar simulator which changes both the equivalent solar concentration in suns as well as the efficiency of the upconverter itself. In the case of the upconverters $\mathrm{Gd}_{2} \mathrm{O}_{2} \mathrm{~S}: 10 \% \mathrm{Er}^{3+}$ and $\beta$-NaYF $\mathrm{Na}_{4}: 25 \% \mathrm{Er}^{3+}$, the $\Delta j_{\text {sc,uc }}$ are shifted to higher values at simultaneously lower solar concentrations due to the spectral mismatch correction.

For the first time, we measured UCSCDs under realistic conditions in an outdoor experiment using sunlight. The corrected solar simulator measurements agree very well with the outdoor measurements. This demonstrates that our spectral mismatch approach can be used to predict the performance of highly non-linear solar devices for measurements performed on wellcontrolled standard solar characterization equipment. 


\section{Acknowledgements}

The research leading to these results has received funding from the European Community's Seventh Framework Programme (FP7/ 2007-2013) under grant agreement no [246200] within the Nanospec project. Stefan Fischer gratefully acknowledges the scholarship support from the German Federal Environmental Foundation (DBU) and the German Research Foundation (DFG, agreement FI 2042/1-1).

\section{References}

(1) Gibart, P.; Auzel, F.; Guillaume, J.-C.; Zahraman, K. Jpn. J. Appl. Phys. 1996, 35 (Part 1, No. 8), 4401.

(2) Trupke, T.; Green, M. a.; Würfel, P. J. Appl. Phys. 2002, 92 (7), 4117.

(3) Trupke, T.; Shalav, A.; Richards, B. S.; Würfel, P.; Green, M. A. Sol. Energy Mater. Sol. Cells 2006, 90 (18-19), 3327.

(4) Shalav, A.; Richards, B. S. B. S.; Trupke, T.; Corkish, R. P.; Krämer, K. W. K. W.; Güdel, H. U.; Green, M. A. M. A.; Güdel, H. U.; Green, M. A. M. A. In 3rd World Conference on Photovoltaic Energy Conversion; 2003; pp 248-250.

(5) Richards, B. S.; Shalav, A. IEEE Trans. Electron Devices 2007, 54 (10), 2679.

(6) Fischer, S.; Goldschmidt, J. C.; Löper, P.; Bauer, G. H.; Brüggemann, R.; Krämer, K.; Biner, D.; Hermle, M.; Glunz, S. W. J. Appl. Phys. 2010, 108 (4), 44912.

(7) Strümpel, C.; McCann, M.; Beaucarne, G.; Arkhipov, V.; Slaoui, A.; Svrcek, V.; del Canizo, C.; Tobias, I. Sol. Energy Mater. Sol. Cells 2007, 91 (4), 238.

(8) de Wild, J.; Rath, J. K.; Meijerink, A.; van Sark, W. G. J. H. M.; Schropp, R. E. I. Sol. Energy Mater. Sol. Cells 2010, 94 (12), 2395.

(9) Huang, X.; Han, S.; Huang, W.; Liu, X. Chem. Soc. Rev. 2013, 42 (1), 173.

(10) Goldschmidt, J. C.; Fischer, S. Adv. Opt. Mater. 2015, 3 (4), 510.

(11) Fischer, S.; Favilla, E.; Tonelli, M.; Goldschmidt, J. C. Sol. Energy Mater. Sol. Cells 2015, 136, 127.

(12) Boccolini, A.; Faoro, R.; Favilla, E.; Veronesi, S.; Tonelli, M. J. Appl. Phys. 2013, 114 (6), 64904.

(13) Rüdiger, M.; Fischer, S.; Frank, J.; Ivaturi, A.; Richards, B. S.; Krämer, K. W.; Hermle, M.; Goldschmidt, J. C. Sol. Energy Mater. Sol. Cells 2014, 128, 57.

(14) Schulze, T. F.; Schmidt, T. W. Energy Environ. Sci. 2015, 8 (1), 103.

(15) Gray, V.; Dzebo, D.; Abrahamsson, M.; Albinsson, B.; Moth-Poulsen, K. Phys. Chem. 
Chem. Phys. 2014, 16 (22), 10345.

(16) Ivaturi, A.; MacDougall, S. K. W.; Martín-Rodríguez, R.; Quintanilla, M.; Marques-Hueso, J.; Krämer, K. W.; Meijerink, A.; Richards, B. S. J. Appl. Phys. 2013, 114 (1), 13505.

(17) Fischer, S.; Fröhlich, B.; Krämer, K. W.; Goldschmidt, J. C. J. Phys. Chem. C 2014, 118 (51), 30106.

(18) Martín-Rodríguez, R.; Fischer, S.; Ivaturi, A.; Froehlich, B.; Krämer, K. W.; Goldschmidt, J. C.; Richards, B. S.; Meijerink, A. Chem. Mater. 2013, 25 (9), 1912.

(19) Fischer, S.; Martín-Rodríguez, R.; Fröhlich, B.; Krämer, K. W.; Meijerink, A.; Goldschmidt, J. C. J. Lumin. 2014, 153, 281.

(20) Boccolini, A.; Marques-hueso, J.; Richards, B. S. Opt. Lett. 2014, 39 (10), 2904.

(21) Auzel, F. Chem. Rev. 2004, 104 (1), 139.

(22) Lüthi, S. R.; Pollnau, M.; Güdel, H. U.; Hehlen, M. P. Phys. Rev. B 1999, 60 (1), 162.

(23) Fischer, S.; Steinkemper, H.; Löper, P.; Hermle, M.; Goldschmidt, J. C. J. Appl. Phys. 2012, $111(1), 13109$.

(24) Goldschmidt, J. C.; Fischer, S.; Löper, P.; Krämer, K. W.; Biner, D.; Hermle, M.; Glunz, S. W. Sol. Energy Mater. Sol. Cells 2011, 95 (7), 1960.

(25) Fischer, S.; Ivaturi, A.; Frohlich, B.; Rudiger, M.; Richter, A.; Kramer, K. W.; Richards, B. S.; Goldschmidt, J. C. IEEE J. Photovoltaics 2014, 4 (1), 183.

(26) Fischer, S.; Fröhlich, B.; Steinkemper, H.; Krämer, K. W.; Goldschmidt, J. C. Sol. Energy Mater. Sol. Cells 2014, 122, 197.

(27) de Wild, J.; Duindam, T. F.; Rath, J. K.; Meijerink, A.; van Sark, W. G. J. H. M.; Schropp, R. E. I. IEEE J. Photovoltaics 2013, 3 (1), 17.

(28) MacDougall, S. K. W.; Ivaturi, A.; Marques-Hueso, J.; Krämer, K. W.; Richards, B. S. Sol. Energy Mater. Sol. Cells 2014, 128, 18.

(29) MacDougall, S. K. W.; Ivaturi, A.; Marques-Hueso, J.; Richards, B. S. Rev. Sci. Instrum. 2014, 85 (6), 63109.

(30) Frank, J.; Rüdiger, M.; Fischer, S.; Goldschmidt, J. C.; Hermle, M. Energy Procedia 2012, $27,300$.

(31) Nitz, P.; Heller, A.; Platzer, W. J. In Proceedings of the 4th International Conference on Solar Concentrators for the Generation of Electricity or Hydrogen; San Lorenzo de el Escorial, 2007; pp 289-292.

(32) Fischer, S. Upconversion of Sub-Band-Gap Photons for Silicon Solar Cells, Dissertation, Albert-Ludwigs-University Freiburg, 2014.

(33) Goldschmidt, J. C.; Löper, P.; Fischer, S.; Janz, S.; Peters, M.; Glunz, S. W.; Willeke, G.; Lifshitz, E.; Kramer, K.; Biner, D. In 2008 Conference on Optoelectronic and Microelectronic Materials and Devices; IEEE, 2008; pp 307-311. 